\title{
Mini-Review: Recent Advances in Aptamer Applications
}

\author{
Gerald Zon*
}

TriLink BioTechnologies, 10770 Wateridge Circle, Suite 200, San Diego, California, 92121 , USA

\section{Article Info}

\section{Article Notes}

Received: October 18, 2020

Accepted: November 12, 2020

\section{*Correspondence:}

Dr. Gerald Zon, TriLink BioTechnologies, 10770 Wateridge Circle, Suite 200, San Diego, California, 92121, USA; Email: gzon@trilinkbiotech.com.

(c) 2020 Zon G. This article is distributed under the terms of the Creative Commons Attribution 4.0 International License.

\section{ABSTRACT}

Aptamers, which are short strands of synthetic DNA or RNA selected for binding to target ligands, have proven useful for cell-specific delivery, detection, or both. This mini-review presents selected examples of these applications for cancer published during 2019 - September 2020.

\section{Introduction}

Aptamers are short (20 to 60 nucleotides) single-stranded DNA or RNA molecules that bind with high affinity and specificity to various ligands. ${ }^{1}$ Aptamers have been referred to as "synthetic antibodies," but are more versatile and can replace antibodies in some applications. ${ }^{2}$ Following two seminal reports ${ }^{3,4}$ in 1990 , published applications of aptamers exceeded 10,000 as of 2019, according to a search of PubMed. ${ }^{5}$ Refinement of this literature, specifically for cancer, gave 1,936 items for 1999 - 2019 (Figure 1). An exponential increase in numbers is evident, as is the likelihood of $\sim 350$ such articles in 2020 alone.

\section{Breast Cancer}

Triple-negative breast cancer (TNBC), an aggressive cancer with

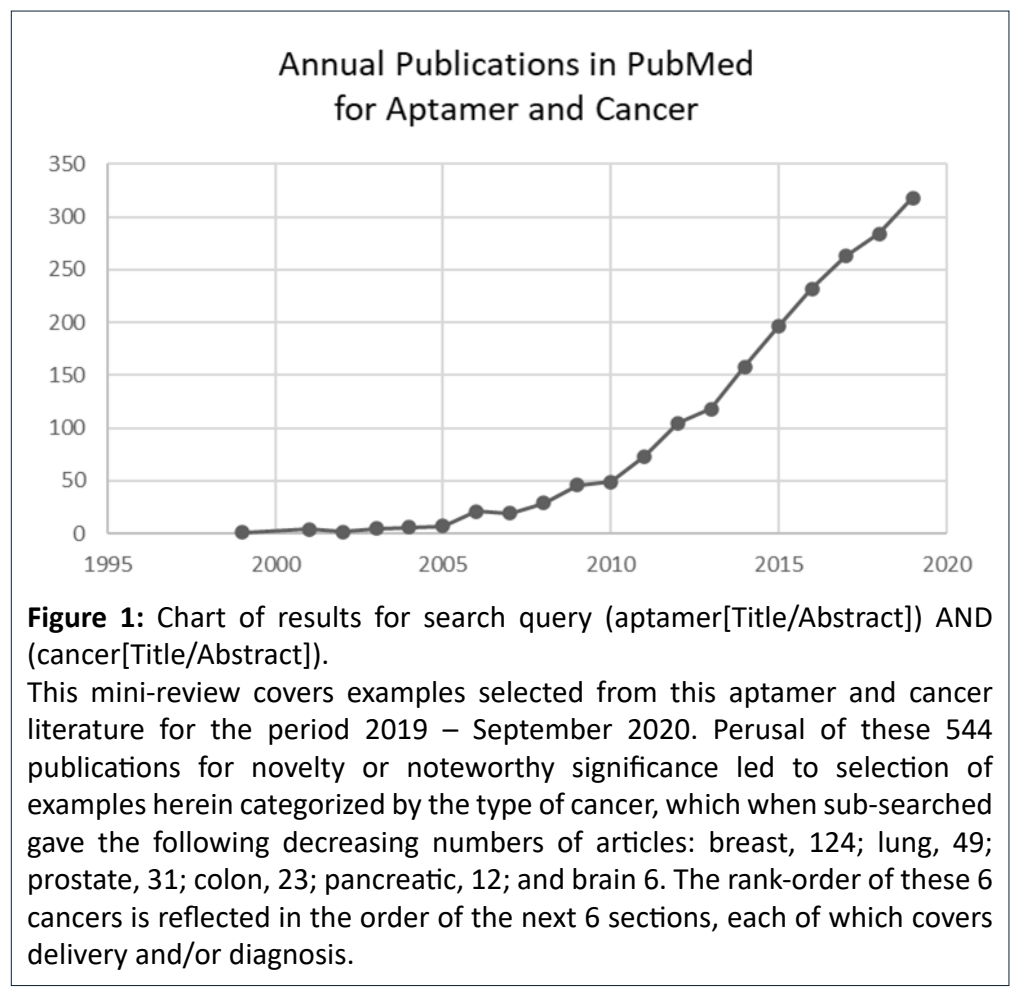


high rates of relapse due to resistance to chemotherapy, expresses higher levels of programmed cell death-ligand 1 (PD-L1) compared to other breast cancers, and thus provides the rationale for FDA-approved immunotherapy with anti-PD-L1 monoclonal antibodies (mAbs). Because platelet-derived growth factor receptor $\beta$ (PDGFR $\beta$ ) is overexpressed by TNBC cells and tumor microenvironment, Camorani et al. ${ }^{6}$ developed a PDGFR $\beta$ aptamer to investigate a novel combination treatment with the PDGFR $\beta$ aptamer and anti-PD-L1 mAbs in TNBC. Importantly, they found that the aptamer increases the antibody efficacy in inhibiting tumor growth and lung metastases in mice by acting on both tumor cells, inhibiting signaling pathways, and increasing intratumoral T cells.

TNBC was also targeted by Yin et al., 7 but using a quite different strategy employing a novel 4-stranded synthetic RNA construct comprised of an aptameric CD133binding region for delivery and a locked nuclei acid (LNA) anti-microRNA, miRNA-21, for inhibition by and RNA interference (RNAi) mechanism. ${ }^{8}$ Mixing the 4 strands provides self-assembled nanoparticles (NPs) for specific uptake by breast cancer stem cells and TNBC cells. Tail vein injections in mice demonstrated highly specific TNBC tumor targeting and significant tumor growth inhibition.

Anti-VCAM-1 and anti-IL4R $\alpha$ DNA aptamers, specific for their cognate receptors overexpressed in 4T1 mammary tumor-bearing mice, were shown by Chinnappan et al. ${ }^{9}$ to be useful for diagnosis and therapy when conjugated to super paramagnetic iron oxide nanoparticles (SPIONs) Receptor binding by both aptamers triggers tumor cell apoptosis, thus limiting tumor progression in vitro and in vivo. Cancer cells expressing the luciferase (Luc2) reporter enzyme, and the negative contrast for magnetic resonance imaging (MRI) induced by SPIONs, allowed noninvasive bioluminescence imaging and MRI, respectively, in 4T1Luc2 tumor-bearing mice, following tail-vein injection.

Effective diagnosis of TNBC using nucleolin (NUC)targeted nanobubbles ${ }^{10}$ for contrast-enhanced ultrasound molecular imaging, reported by Fang et al., ${ }^{11}$ is based on the overexpression of NUC by endothelial cells of breast tumor blood vessels. The well-known anti-NUC aptamer AS1411 was conjugated to a lipid that was then used to form the nanobubbles. Imaging results obtained with these nanobubbles in vivo demonstrated prolonged duration of NUC-expressing neovasculature compared to control nanobubbles.

Finally, a wide variety of non-lipid nanoparticles have been developed for aptamer-based sensors (aptasensors) that use optical and electrochemical strategies for detection, as reviewed in 2020 by Mao et al. ${ }^{12}$

\section{Lung Cancer}

Anti-NUC aptamer AS1411 has also been used by Nie et al. ${ }^{13}$ to target the 4549 human lung carcinoma cell line by coloading, with the drug doxorubicin, onto poly(ethylenimine) grafted with polyethylene glycol copolymer functionalized gold nanoparticles through amide reactions.

A novel synthetic RNA construct comprised of an aptameric region for delivery and a miRNA for RNAi was reported by Nuzzo et al. ${ }^{14}$ as a new therapeutic approach for non-small-cell lung cancer (NSCLC), which accounts for $\sim 85 \%$ of all lung cancers, is quite deadly, and warrants new treatment options. The 2 '-fluoropyrimidine-modified nuclease-resistant RNA aptamer, termed GL21.T, binds to and antagonizes the oncogenic receptor tyrosine kinase Axl, while miRNA-137 targets several genes known to play important roles in various human cancers and cell-cycle signaling. The A549 cell line used for studies of lung cancer, when treated with the chimeric construct, GL21.T-137, showed significantly increased levels of miRNA-137 by qRTPCR, compared to controls. The levels of Cdk6, a validated miRNA-137 target, were comparatively reduced by $40 \%$ at $48 \mathrm{~h}$. Moreover, inhibition of cell migration and growth by GL21.T-137 was shown for $\mathrm{Axl}^{+}$primary cells obtained from surgical specimens from NSCLC patients, and there was significant reduction in tumor volume in a A549 mouse xenograft models following 7 days intraperitoneal injection of GL21.T-137, compared to controls.

Selective eradication of human NSCLC cells using aptamer-decorated NPs loaded with a cytotoxic drug was described by Engelberg et al. ${ }^{15}$ The NPs, comprised of biocompatible polyethylene-glycol (PEG) conjugated to polycaprolactone (PCL), PEG-PCL, self-assembled in the presence of paclitaxel (PTX), and were then conjugated to S15-APT, a DNA aptamer previously selected for specific binding to NSCLC by others. ${ }^{16}$ Confocal microscopy using NPs having dye-labeled S15-APT demonstrated internalization by A549 cells but not normal human bronchial epithelial BEAS2B cells, cervical carcinoma HeLa, colon adenocarcinoma CaCo-2, neonatal foreskin fibroblast FSE, and human embryonic kidney HEK-293 cells. Notably, the selective cytotoxicity of these NPs toward A549 cells was reflected in the $\mathrm{IC}_{50}$ value, which was approximately 50- to 30,000 -fold lower than those for these other cell types.

An example of an aptamer outperforming an antibody was provided by Zhang et al., ${ }^{17}$ according to whom conventional antibody-based immunocytochemistry (ICC) on cytological samples can sometimes be questionable. To address these limitations in the context of lung cancer, they developed ICC diagnosis employing S6, a DNA aptamer specific for A549 lung carcinoma cells reported by others. ${ }^{18}$ Three different formats for S6 were investigated: biotinlabeled, fluorescent FAM-labeled, and conformationally activatable. A total of 112 serosal fluid specimens with known diagnoses were evaluated by all three strategies. 
ICC procedures based on biotin-labeled or FAM-labeled S6 aptamers required time-consuming washing to avoid interference from nonspecific adsorption. By contrast, the activatable ICC format showed strong fluorescence in the presence of target cells without any washing steps. Importantly, the specificity and sensitivity were found to be higher in all three of these S6 aptamer-based ICC protocols relative to those for antibody protocols for differentiating adenocarcinoma cells in clinical effusion cytology.

\section{Prostate Cancer}

Like the use of SPIONs for breast cancer, ${ }^{9}$ these magnetic NPs were studied by Fang et al. ${ }^{19}$ using the known prostate cancer (PC)-specific aptamer, Wy5a, for delivery of Docetaxel (Dtxl), a broad-spectrum anticancer drug. These targeted SPIONs showed controlled drug-release in PC-3 cells in vitro and in vivo, as well as MRI contrastenhancement, plus more efficacious antitumor properties in vivo without systemic toxicity.

In view of $\mathrm{NIH}$ fostering research in alternative medicine, ${ }^{20}$ it is worth mentioning two studies of aptamerguided delivery of curcumin (CUR), a natural product derived from the spice turmeric, the health effects of which have been reviewed elsewhere. ${ }^{21}$ In the first study, Ma et al. ${ }^{22}$ prepared conventional nanoliposomes (NLPs) that were then conjugated to an RNA aptamer, A15, known to bind to CD133, which marks normal prostate epithelial stem cells and rare malignant tumor stem/initiating cells. The NLPs were loaded with CUR to provide A15-CUR-NLPs for which drug burst-release was observed within $2 \mathrm{~h}$, followed by continuous release over $48 \mathrm{~h}$. In vitro comparisons of A15CUR-NLPs with CUR-NLPs in DU145 cells showed greater uptake and cytotoxicity for the former. DU145 xenograft mice bearing tumors were divided into four groups $(n=6)$ : (1) free CUR (25 $\mu \mathrm{g}$ in DMSO), (2) CUR-NLPs (25 $\mu \mathrm{g}$ CUR equivalent in PBS), (3) A15-CUR-NLPs (25 $\mu \mathrm{g}$ CUR equivalent in PBS), and (4) empty NLPs. Compared to 4 , relative tumor inhibition was $1=45 \%, 2=57 \%$ and $3=82 \%$.

The second study by Chen et al. ${ }^{23}$ employed an RNA aptamer (APT), which was modified with 2 '-fluoropyrimidines for nuclease resistance. ${ }^{14}$ LNPs with conjugated APT were loaded with CUR and cabazitaxel (Ctx), a microtubule inhibitor approved for hormone-refractory PC. The APT-CUR/Ctx-LPNs exhibited sustained release of both CUR and Ctx. In vitro assays demonstrated synergistic cytotoxicity. PC xenograft mice were randomized into 7 groups $(n=6)$ and intravenously injected with (1) APTCUR/Ctx-LPNs, (2) APT-CUR-LPNs, (3) APT-CTX-LPNs, (4) CUR/Ctx-LPNs, (5) CUR and CTX mixed solution, (6) APTLPNs, and (7) saline (NS) every 3 days at an equivalent dose of CUR ( $2 \mathrm{mg} / \mathrm{kg}$ ) and/or CTX (5 mg/kg). After 21 days, tumor volumes indicated that inhibition for 1 was 2.5-times greater than those for 2,3 , or 4 , which were significantly greater than that for 5-7.

Most of the 31 recent aptamer/PC publications were new methods to detect prostate specific antigen (PSA) in blood, the current gold standard in prostate screening. A completely different diagnostic approach was described by Li et al., ${ }^{24}$ who investigated the contents of exosomes (30 $150 \mathrm{~nm}$ extracellular vesicles) in urine derived by prostate cells. SPIONs ${ }^{9}$ were decorated with an aptamer for prostate specific membrane antigen (PSMA) for magnetic capture of PSMA-positive exosomes in urine, thus avoiding invasive blood collection. This was followed by a nonenzymatic oligonucleotide amplification cycle using dye-labeled molecular beacons, ${ }^{25}$ and exosome isolation using a restriction enzyme to remove SPIONs. The beacons gave a limit of detection of $\sim 100$ exosome particles/ $\mu \mathrm{L}$ of urine, and fluorescence intensity significantly differentiated PC vs. normal patient samples. Recovered exosomes were subjected to qRT-PCR to detect the mRNAs for PSA and prostate cancer antigen 3 (PCA3) from a gene that expresses a non-coding RNA. PCA3 is only expressed in human prostate tissue, and the gene is highly overexpressed in prostate cancer, which makes it an ideal biomarker. The PSA and PCA3 levels significantly correlated PC vs. normal patient samples.

\section{Colon Cancer}

Based on their previous work that identified miRNA139-5p as a tumor suppressor gene that inhibits progression of colorectal cancer (CRC), Zhao et al. ${ }^{26}$ developed a LNP loaded with miRNA-139-5p that is decorated with an epithelial cell adhesion molecule (EpCAM) aptamer for targeted treatment of CRC. These LNPs were taken up by multiple CRC cell lines in vitro and markedly inhibited proliferation, including HCT8 cells, proliferation of which was significantly inhibited, subcutaneously, in a mouse tumor model.

Babaei et al. ${ }^{27}$ investigated simultaneous drug and gene delivery to colorectal adenocarcinoma as a means of obtaining synergistic effects. An AS1411 DNA NUC $^{11}$-targeted co-delivery system, based on PEGylated mesoporous silica NPs, was developed for delivery of camptothecin (CPT), a topoisomerase inhibitor, and a commercially available apoptosis-inducing ${ }^{28}$ survivin shRNA-expressing plasmid, iSur-DNA. Controlled release of CPT was demonstrated, as was potent synergistic cytotoxicity and apoptosis in vitro. The NUC aptamer facilitated drug uptake into $\mathrm{NUC}^{+} \mathrm{C} 26$ colorectal cancer cells leading to higher cellular toxicity and apoptosis compared to NUC- $\mathrm{CHO}^{-}$cells. The aptamer-guided NPs suppressed tumor growth in C26 tumor-bearing mice and improved survival, compared to controls. 


\section{Pancreatic Cancer}

According to Rossi \& coworkers, ${ }^{29}$ pancreatic ductal adenocarcinoma (PDAC) is one of the most aggressive malignancies; it preferentially metastasizes to the liver and is the main cause of death from this disease. In their previous studies, a small activating RNA (saRNA) - an emerging therapeutic strategy for transcriptional gene activation - against CCAAT/enhancer-binding protein- $\alpha$ (C/EBP $\alpha$-saRNA) was efficacious in a PDAC tumor model. The present work, focused on advanced stage PDAC, employed a new anti-transferrin receptor aptamer, TR14, conjugated to $\mathrm{C} / \mathrm{EBP} \alpha$-saRNA. Treatment of pancreatic cancer cells with the conjugate upregulated expression of C/EBP $\alpha$ and its downstream target $\mathrm{p} 21$, and inhibited cell proliferation. in an advanced PDAC mouse model, treatment with TR14-C/EBP $\alpha$-saRNA conjugate led to significant reduction of tumor growth.

To address the need for early detection of pancreatic cancer, Zou et al. $^{30}$ explored the feasibility of using MRI in mucin 1 (MUC1)-conjugated SPIONs ${ }^{9}$ (MUC1-SPIONs) targeting human pancreatic cancer. The absence of toxicity of MUC1-SPIONs was demonstrated by the standard MTS assay, using BxPC-3 cells, for which a nude mouse model of pancreatic cancer was established to investigate imaging. MRI was performed to determine the intensity of the signal of the transplanted tumor, while immunohistochemistry and Western blot analysis were used to detect the expression of MUC1 after removal of the transplanted tumor. Importantly, MUC1 selectively accumulated in the tumor and significantly reduced the $\mathrm{T}_{2}$-MRI signal strength. In related experiments, expression of MUC1 was high in pancreatic cancer tissues, but low in normal pancreatic tissues.

\section{Brain Cancer}

Affinito et al. ${ }^{31}$ state that glioblastoma (GBM) is the most aggressive primary brain tumor in adults and, despite progress in surgical and medical neuro-oncology, prognosis for GBM patients remains dismal, with a median survival of only 14 - 15 months. This due to residual GBM stem cells (GSCs) that cause tumor relapse and chemoresistance. Because strategies to identify and target GSCs have been unsuccessful, these researchers developed, for first time, an RNA aptamer, A40s, that selectively binds GSCs vs. undifferentiated GBM cells and is rapidly internalized. Feasibility for delivery was demonstrated first using stickyend annealing of A40s to miRNA-34c, a p53 effector, which has antiproliferative and pro-apoptotic functions. This annealing was next used for an anti-miRNA-10b sequence to knock-down miRNA-10b, a miRNA not expressed in human brain and strongly up-regulated in both low-grade and high-grade gliomas.

\section{Conclusions}

Despite the small "sample size" of these selected examples, it is evident that aptamer-enabled delivery for cancer is applicable to a remarkably broad spectrum of therapeutic agents and detection schemes. This is especially true for aptamers attached to nanoparticles, which can accommodate small-molecule drugs, synthetic oligonucleotide therapeutics of all mechanistic types, and/or large gene-encoded plasmids. While not covered here, this diverse cargo has been extended to geneediting strategies, notably CRISPR, ${ }^{32}$ the revolutionary genetic "scissors," honored by the 2020 Chemistry Nobel Prize shared by Emmanuelle Charpentier and Jennifer A. Doudna. Indeed, 10 such papers can already be found by searching PubMed for aptamer, cancer, and CRISPR in the Title/Abstract.

The future prospects for treatment and diagnosis of cancer using aptamers looks very promising indeed, given the huge number of possible combinations comprised of aptamers for specific targeting, ancillary agents for therapy and/or diagnosis, and nanoparticle materials for delivery and/or detection. This vast array of tools, coupled with the imagination and excitement of dedicated researchers, point toward many major advances against cancer yet to come, which is a perspective also opined by others in a very recent review. ${ }^{33}$

\section{References}

1. Lakhin AV, Tarantul VZ,Gening LV. Aptamers: problems, solutions and prospects. Acta Naturae. 2013; 5: 34-43.

2. Miersch S, Sidhu SS. Synthetic antibodies: concepts, potential and practical considerations. Methods. 2012; 57: 486-498.

3. Ellington AD,Szostak JW. In vitro selection of RNA molecules that bind specific ligands. Nature. 1990; 346: 818-822.

4. Tuerk C, Gold L. Systematic evolution of ligands by exponential enrichment: RNA ligands to bacteriophage T4 DNA polymerase. Science. 1990; 249: 505-510.

5. Zon G. Recent advances in aptamer applications for analytical biochemistry. Anal Biochem. 2020: 113894.

6. Camorani S, Passariello M, Agnello L, et al. Aptamer targeted therapy potentiates immune checkpoint blockade in triple-negative breast cancer. Journal of experimental \& clinical cancer research : CR. 2020; 39: $180-180$

7. Yin H, Xiong G, Guo S, et al. Delivery of Anti-miRNA for Triple-Negative Breast Cancer Therapy Using RNA Nanoparticles Targeting Stem Cell Marker CD133. Molecular therapy : the journal of the American Society of Gene Therapy. 2019; 27: 1252-1261.

8. Wilson RC,Doudna JA. Molecular Mechanisms of RNA Interference. Annual Review of Biophysics. 2013; 42: 217-239.

9. Chinnappan R, Al Faraj A, Abdel Rahman AM, et al. Anti-VCAM-1 and Anti-IL4R $\alpha$ Aptamer-Conjugated Super Paramagnetic Iron Oxide Nanoparticles for Enhanced Breast Cancer Diagnosis and Therapy. Molecules (Basel, Switzerland). 2020; 25: 3437.

10. Alheshibri M, Qian J, Jehannin M, et al. A History of Nanobubbles. Langmuir. 2016; 32: 11086-11100. 
11. Fang K, Wang L, Huang H, et al. Construction of Nucleolin-Targeted Lipid Nanobubbles and Contrast-Enhanced Ultrasound Molecula Imaging in Triple-Negative Breast Cancer. Pharm Res. 2020; 37: 145.

12. Mao K, Zhang H, Wang Z, et al. Nanomaterial-based aptamer sensors for arsenic detection. Biosensors and Bioelectronics. 2020; 148: 111785.

13. Nie L, Sun S, Sun M, et al. Synthesis of Aptamer-PEI-g-PEG Modified Gold Nanoparticles Loaded with Doxorubicin for Targeted Drug Delivery. JoVE. 2020: e61139.

14. Nuzzo S, Catuogno S, Capuozzo M, et al. Axl-Targeted Delivery of the Oncosuppressor miR-137 in Non-small-Cell Lung Cancer. Mol Ther Nucleic Acids. 2019; 17: 256-263.

15. Engelberg S, Netzer E, Assaraf YG, et al. Selective eradication of human non-small cell lung cancer cells using aptamer-decorated nanoparticles harboring a cytotoxic drug cargo. Cell Death Dis. 2019; 10: 702.

16. Zhao Z, Xu L, Shi X, et al. Recognition of subtype non-small cell lung cancer by DNA aptamers selected from living cells. Analyst. 2009; 134: 1808-1814.

17. Zhang Y, Xu J, Li D, et al. Immunocytochemistry Based on a Cell-TypeSpecific Aptamer for Rapid Immunostaining of Adenocarcinoma Cells in Clinical Serosal Fluids. Pathol Oncol Res. 2019; 25: 1143-1152.

18. Shi H, Cui W, He X, et al. Whole cell-SELEX aptamers for highly specific fluorescence molecular imaging of carcinomas in vivo. PLoS One. 2013; 8: e70476.

19. Fang Y, Lin S, Yang F, et al. Aptamer-Conjugated Multifunctional Polymeric Nanoparticles as Cancer-Targeted, MRI-Ultrasensitive Drug Delivery Systems for Treatment of Castration-Resistant Prostate Cancer. Biomed Res Int. 2020; 2020: 9186583.

20. Harlan WR, Jr. New opportunities and proven approaches in complementary and alternative medicine research at the National Institutes of Health. J Altern Complement Med. 2001; 7 Suppl 1: S53-59.

21. Hewlings SJ,Kalman DS. Curcumin: A Review of Its Effects on Human Health. Foods (Basel, Switzerland). 2017; 6: 92.

22. Ma Q Qian W, Tao W, et al. Delivery Of Curcumin Nanoliposomes Using Surface Modified With CD133 Aptamers For Prostate Cancer. Drug Des Devel Ther. 2019; 13: 4021-4033.
23. Chen Y, Deng Y, Zhu C, et al. Anti prostate cancer therapy: Aptamerfunctionalized, curcumin and cabazitaxel co-delivered, tumor targeted lipid-polymer hybrid nanoparticles. Biomedicine \& Pharmacotherapy. 2020; 127: 110181.

24. Li P, Yu X, Han W, et al. Ultrasensitive and Reversible Nanoplatform of Urinary Exosomes for Prostate Cancer Diagnosis. ACS Sensors. 2019; 4: 1433-1441.

25. Tyagi S, Kramer FR. Molecular Beacons: Probes that Fluoresce upon Hybridization. Nature Biotechnology. 1996; 14: 303-308.

26. Zhao Y, Xu J, Le VM, et al. EpCAM Aptamer-Functionalized Cationic Liposome-Based Nanoparticles Loaded with miR-139-5p for Targeted Therapy in Colorectal Cancer. Mol Pharm. 2019; 16: 4696-4710.

27. Babaei M, Abnous K, Mohammad Taghdisi S, et al. Targeted rod-shaped mesoporous silica nanoparticles for the co-delivery of camptothecin and survivin shRNA in to colon adenocarcinoma in vitro and in vivo. Eur J Pharm Biopharm. 2020.

28. Xu JH, Wang AX, Huang HZ, et al. Survivin shRNA induces caspase-3dependent apoptosis and enhances cisplatin sensitivity in squamous cell carcinoma of the tongue. Oncol Res. 2010; 18: 377-385.

29. Yoon S, Huang KW, Andrikakou P, et al. Targeted Delivery of C/EBP $\alpha$ saRNA by RNA Aptamers Shows Anti-tumor Effects in a Mouse Model of Advanced PDAC. Molecular therapy. Nucleic acids. 2019; 18: $142-$ 154.

30. Zou Q Zhang CJ, Yan YZ, et al. MUC-1 aptamer targeted superparamagnetic iron oxide nanoparticles for magnetic resonance imaging of pancreatic cancer in vivo and in vitro experiment. J Cell Biochem. 2019; 120: 18650-18658.

31. Affinito A, Quintavalle C, Esposito CL, et al. The Discovery of RNA Aptamers that Selectively Bind Glioblastoma Stem Cells. Molecular therapy. Nucleic acids. 2019; 18: 99-109.

32. Jinek M, Chylinski K, Fonfara I, et al. A Programmable Dual-RNAGuided DNA Endonuclease in Adaptive Bacterial Immunity. Science. 2012; 337: 816-821.

33. Han J, Gao L, Wang J, et al. Application and development of aptamer in cancer: from clinical diagnosis to cancer therapy. J Cancer. 2020; 11: 6902-6915. 